\title{
Dissecção Causando Estenose de Artéria de Rim Transplantado: Diagnóstico por Angiografia Rotacional Tridimensional
}

Erlon Oliveira de Abreu-Silva ${ }^{1}$, Rodrigo Almeida de Souza ${ }^{1}$, Fábio Rodrigo Furini ${ }^{1}$, Adriano Henrique Pereira Barbosa', Claudia Maria Rodrigues Alves' ${ }^{1}$, Valter Correia de Lima'

\section{RESUMO}

Paciente do sexo masculino, com 47 anos de idade, com diagnóstico de doença renal crônica estágio 5 por doença renal policística e em terapia dialítica por cinco anos foi submetido a transplante renal. No pós-operatório, o paciente apresentou disfunção do enxerto e hipertensão não-controlada, havendo necessidade de reintrodução da diálise. Ultrassom Doppler da artéria de rim transplantado foi sugestiva de estenose, e angiotomografia computadorizada confirmou o achado. No 49 dia de pós-operatório, foi realizada angiografia rotacional tridimensional da artéria do enxerto renal e visualizou-se imagem de dissecção causando estenose grave. Foi realizada angioplastia com implante de stent com sucesso e o paciente não mais necessita de diálise e mantém boa evolução do quadro nos últimos seis meses. Estenose de artéria renal é complicação comum, secundária, na maioria das vezes, a placa aterosclerótica. Rastreamento com ultrassom Doppler e confirmação diagnóstica com angiografia são as estratégias recomendadas, visando à intervenção. Dissecção é uma possível causa de estenose de artéria de rim transplantado.

DESCRITORES: Transplante de rim. Dissecção. Obstrução da artéria renal. Angioplastia. Stents.

E stenose de artéria de rim transplantado é uma complicação frequente e importante, cuja incidência varia entre $1 \%$ e $23 \% .^{1,2}$ Pode causar disfunção do enxerto e/ou hipertensão não controlada e colocar o paciente em risco de retornar ao tratamento com diálise.

\footnotetext{
${ }^{1}$ Universidade Federal de São Paulo (Unifesp) - São Paulo, SP, Brasil.

Correspondência: Erlon Oliveira de Abreu-Silva. Rua Borges Lagoa, 1.209/1.809 - Vila Clementino - São Paulo, SP, Brasil - CEP 04038-033 E-mail: erlonsilva@yahoo.com.br

Recebido em: 3/9/2011 • Aceito em: 9/11/2011
}

\begin{abstract}
Dissection Causing Transplant Renal Artery Stenosis: Diagnosis by 3D Rotational Angiography

A 47-year-old man diagnosed with stage 5 chronic kidney disease due to polycystic kidneys and on dialysis for five years was submitted to kidney transplantation. During the post-operative period the patient presented with graft dysfunction and uncontrolled hypertension, requiring reintroduction of dialysis. Doppler ultrasound suggested stenosis of the transplant renal artery, which was confirmed by CT angiography. On the $49^{\text {th }}$ day after surgery, 3D rotational angiography of the renal graft artery was performed, showing dissection causing severe stenosis. Angioplasty and stent implantation were successfully performed and the patient no longer requires dialysis and has evolved well in the past six months. Transplant renal artery stenosis is a common complication, and is secondary to atherosclerotic plaque in most of the cases. Screening with Doppler ultrasound and diagnostic confirmation by angiography are the recommended strategies for intervention. Dissection is a possible cause of transplant renal artery stenosis.
\end{abstract}

KEY-WORDS: Kidney transplantation. Dissection. Renal artery obstruction. Angioplasty. Stents.

A doença aterosclerótica é a causa mais comum de estenose, mas dissecção, espontânea ou secundária a manipulação, é também descrita como um mecanismo fisiopatológico de comprometimento da luz em vasos nativos, inclusive de artérias renais.

Ultrassom Doppler é o método de escolha para triagem de estenose de artéria de rim transplantado, enquanto o diagnóstico definitivo é feito por angiografia. Entretanto, em alguns casos, a angiografia convencional não provê toda a informação necessária sem o uso de projeções não-convencionais e, consequentemente, maiores quantidades de contraste iodado e de exposição a radiação ionizante. A angiografia rotacional tridimensional (AR-3D) pode ser alternativa eficaz, oferecendo uma visão espacial mais completa com menor 
volume de contraste e de exposição à radiação. Essa informação é importante no planejamento da estratégia para o procedimento terapêutico.

Os autores descrevem o caso de um paciente submetido a AR-3D para avaliação de estenose de artéria de rim transplantado como a causa de disfunção de enxerto, na qual a dissecção da artéria do transplante foi identificada e tratada com sucesso por meio do implante de stent.

\section{RELATO DO CASO}

Paciente do sexo masculino, com 47 anos de idade, com insuficiência renal crônica estágio 5 decorrente de rins policísticos e que esteve em terapia dialítica por cinco anos, foi submetido a transplante renal. Não houve complicações intraoperatórias. Entretanto, o paciente apresentou sinais de disfunção do enxerto durante a hospitalização, tais como níveis elevados de escórias nitrogenadas e hipertensão não-controlada, sendo necessária a reintrodução de diálise.

Foi realizado ultrassom Doppler da artéria de enxerto renal, que demonstrou pico de velocidade sistólica de $663 \mathrm{~cm} / \mathrm{s}$, sugerindo estenose. Angiotomografia computadorizada demonstrou artéria renal transplantada única anastomosada à artéria ilíaca externa direita, com estenose focal em sua porção proximal. O paciente foi submetido a AR-3D da artéria de rim transplantado no 49ํㅡㄹ dia de pós-operatório.

O protocolo de nossa instituição para a realização da AR-3D, já descrito em publicação prévia, utiliza um equipamento Philips Allura ${ }^{\mathrm{TM}}$ FD10 (Philips \& Co., Eindhoven, Holanda) e um software dedicado, com configurações predefinidas para aquisição e manuseio de imagens do abdômen. Após acesso percutâneo à artéria femoral direita, um cateter pigtail é posicionado na artéria ilíaca externa direita proximal ao óstio da artéria de rim transplantado e a mesa de exame é posicionada centrando o enxerto e seus vasos em duas projeções (posterior/anterior neutra $-0 \% 0^{\circ}$; e oblíqua anterior esquerda neutra $-90^{\circ} / 0^{\circ}$ ). Automaticamente, as posições inicial e final para a rotação do arco são obtidas a $90^{\circ}$ e $-90^{\circ}\left(180^{\circ}\right)$ e 120 imagens são adquiridas em 4,1 segundos, usando $40 \mathrm{ml}$ de meio de contraste iodado (Hexabrix ${ }^{\text {TM }} 320$ mg I/ml, Grupo Guerbet, Villepinte, França) injetados a $10 \mathrm{ml} / \mathrm{s}$, com 1,5 segundo de retardo, usando bomba de injeção (Medrad ${ }^{\mathrm{TM}}$ Mark $\checkmark$ Plus, Medrad Inc., Pittsburgh, Estados Unidos).

O exame mostrou imagem sugestiva de dissecção causando estenose significativa da porção proximal da artéria do enxerto renal (Figura 1). Foi realizada angioplastia com implante de stent Dynamic Renal ${ }^{\mathrm{TM}}$ 7,0 x 12 mm (Biotronik, Berlin, Alemanha), com sucesso angiográfico e sem complicações (Figura 2).

O paciente apresentou melhora da função renal e do controle da pressão arterial, tendo recebido alta

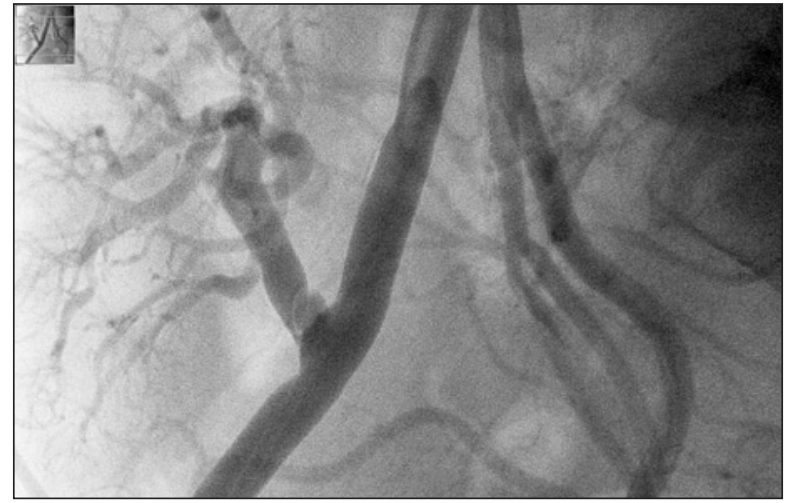

Figura 1 - Angiografia demonstrando o aspecto inicial da dissecção espontânea na porção proximal da artéria de rim transplantado.

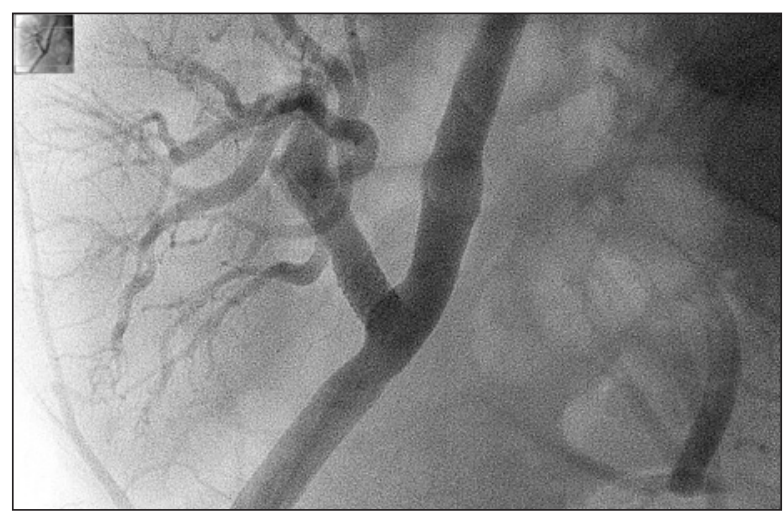

Figura 2 - Angiografia demonstrando o aspecto final da artéria de rim transplantado após implante de stent e resolução da dissecção.

hospitalar dois dias depois do procedimento. A diálise foi suspensa 30 dias após a intervenção. Ultrassom Doppler realizado 60 dias após o implante do stent não mostrou sinais de estenose da artéria de rim transplantado.

\section{DISCUSSÃO}

A doença renal crônica causa grande impacto no sistema público de saúde em virtude de sua alta incidência e prevalência ${ }^{4}$, e o mesmo é verdade para suas condições predisponentes mais frequentes, tais como hipertensão arterial e diabetes melito. ${ }^{4}$

Sabe-se que esses pacientes estão sujeitos a maior risco cardiovascular e que o processo aterosclerótico é acelerado naqueles em tratamento com diálise. ${ }^{5} \mathrm{O}$ transplante renal diminui o risco e aumenta a sobrevida dessa população. ${ }^{6}$

A incidência de estenose de artéria de rim transplantado, a principal complicação do transplante renal, varia entre $1 \%$ e $23 \%{ }^{1,2}$, sendo a principal causa de disfunção do enxerto e/ou de hipertensão não-controlada nesses casos. Diagnóstico precoce e detalhamento anatômico 
preciso são de fundamental importância para o planejamento e a execução do tratamento. Ultrassom Doppler é o exame de triagem mais comum e de melhor relação custo-efetividade ${ }^{7}$, embora também possam ser utilizadas a angiotomografia computadorizada e a ressonância magnética. A angiografia da artéria renal é o padrão de referência, permitindo a quantificação das dimensões da artéria e do estreitamento luminal, bem como a medida do gradiente de pressão. ${ }^{8,9} \mathrm{~A}$ angiografia tridimensional rotacional demonstrou resultados promissores na radiologia e na neurologia intervencionista, e, recentemente, Souza et al. ${ }^{3}$ relataram a experiência da Universidade Federal de São Paulo/Unifesp (São Paulo, SP) com a AR-3D na avaliação da estenose de artéria de rim transplantado.

A placa aterosclerótica é a causa mais comum de estenose arterial, mas a dissecção é também descrita como um mecanismo fisiopatológico de comprometimento da luz em vasos nativos, incluindo artérias renais. $\mathrm{Na}$ estenose de artéria de rim transplantado, aterosclerose, fatores relacionados à técnica cirúrgica e também dissecção espontânea são causas descritas. ${ }^{10}$

A intervenção percutânea e o implante de stent demonstraram ser eficazes no tratamento da estenose de artéria de rim transplantado, com melhora da função renal e do controle da pressão arterial. ${ }^{10}$

Um aspecto importante neste relato é o uso de uma nova tecnologia, que se mostrou eficaz no detalhamento da anatomia da lesão, no planejamento e na execução do tratamento percutâneo, com projeções mais reveladoras, menor volume de contraste e menores doses de radiação.

Em conclusão, a estenose de artéria de rim transplantado deve sempre estar na lista de hipóteses diagnósticas de um paciente que se apresente com disfunção do enxerto e/ou hipertensão não-controlada após transplante renal. O ultrassom Doppler é o exame de triagem mais disponível e de melhor relação custo-efetividade e a AR-3D apresenta bons resultados no diagnóstico e no tratamento desses casos. Embora a placa aterosclerótica seja a causa mais comum de estenose de artéria de rim transplantado, dissecção é um possível mecanismo como nos vasos nativos - e o tratamento percutâneo demonstra bons resultados angiográficos e clínicos.

\section{CONFLITO DE INTERESSES}

Os autores declaram não haver conflito de interesses relacionado a este manuscrito.

\section{REFERÊNCIAS}

1. Hurst FP, Abbot KC, Neff RT, Elster EA, Falta EM, Lentine $\mathrm{KL}$, et al. Incidence, predictors and outcomes of transplant renal artery stenosis after kidney transplantation: analysis of USRDS. Am J Nephrol. 2009;30(5):459-67.

2. Fervenza FC, Lafayette RA, Alfrey EJ, Petersen J. Renal artery stenosis in kidney transplants. Am J Kidney Dis. 1998;31(1): 142-8.

3. Souza RA, Barbosa AHP, Pimentel LC, Furini FR, Sousa JMA, Medina-Pestana JO, et al. Angiografia rotacional com reconstrução tridimensional (3D-RA) como ferramenta diagnóstica em pacientes com estenose da artéria do rim transplantado. Rev Bras Cardiol Invasiva. 2011;19(1):78-83.

4. United States Renal Data System. USRDS 2007 Annual Data Report: Atlas of Chronic Kidney Disease and End-Stage Renal Disease in the United States [Internet]. Bethesda (MD): National Institutes of Health, National Institute of Diabetes and Digestive and Kidney Diseases; 2007 [cited 2011 May 15] Available from: http://www.usrds.org/adr.htm

5. Amann K, Ritz C, Adamczak M, Ritz E. Why is coronary heart disease of uremic patients so frequent and so devastating? Nephrol Dial Transplant. 2003;18(4):632-40.

6. Rambod M, Shabani M, Shokrpour N, Rafii F, Mohammadalliha J. Quality of life of hemodialysis and renal transplantation patients. Health Care Manag (Frederick). 2011;30(1):23-8.

7. Jakobsen JA, Brabrand K, Egge TS, Hartmann A. Doppler examination of the allografted kidney. Acta Radiol. 2003; 44(1):3-12.

8. Hofmann LV, Smith PA, Kuszyk BS, Kraus E, Fishman EK. Three-dimensional helical CT angiography in renal transplant recipients: a new problem-solving tool. AJR Am J Roentgenol. 1999;173(4):1085-9.

9. Neimatallah MA, Dong Q, Schoenberg SO, Cho KJ, Prince MR. Magnetic resonance imaging in renal transplantation. J Magn Reson Imaging. 1999;10(3):357-68.

10. Humke U, Takahashi M, Siemer S, Uder M. Interventional radiologic management for early post-transplant perfusion failure of renal allografts. Aktuelle Urol. 2004;35(4):320-5. 